\title{
Fermionic response in nonlinear arcsin electrodynamics
}

\author{
Nishal Rai ${ }^{\mathrm{a}}$ \\ SRM University Sikkim, 5th Mile, East Sikkim, Gangtok 737102, India
}

Received: 30 September 2019 / Accepted: 7 November 2019 / Published online: 26 November 2019

(C) The Author(s) 2019

\begin{abstract}
We consider certain blackhole solution in nonlinear arcsin electrodynamics coupled with gravity and axions. We have studied the behaviour of the fermionic operators in the dual (2+1)-dimensional theory. We consider holographic spectral function for both the backreacted solutions and probe limit over the range of physical parameters. We find that with a variation of the charge density the system changes from Fermi liquid to non-Fermi liquid and the transition point depends on the temperature.
\end{abstract}

\section{Introduction}

Strongly coupled systems arose in condensed matter turns out to be suitable arena [1-8] for application of holographic techniques of gauge/Gravity duality [9-12]. These techniques can address strongly coupled non-gravitational systems in the set up of a gravity theory with a black hole background. In particular, analysis of fermionic excitations [13-15] exhibits scaling behaviour of non-Fermi liquids in this set up. Further studies regarding scaling exponents and dimension of dual theories appear in [16]. Introduction of dipole coupling on the gravity side leads to dynamically generated gap $[17,18]$. Charged Lifshitz black branes were considered in $[19,20]$. Effect of doping parameters on fermionic excitations in the holographic set up was studied in $[21,22]$ and gives rise to transition between Fermi liquid to non-Fermi liquid phase. Similar studies of fermions in top down approach appear in [23-28].

All these studies involve Maxwell electrodynamics in the gravity setup. Even though these linear electrodynamic models are successful in explaining various features in the context of condensed matter systems, it fails to capture the behaviour of some of the systems, one such is cuprates high $T_{c}$ superconductors in the strange metal phase where the resistivity depends linearly on temperature and so called the anoma-

\footnotetext{
a e-mail: nishalrai10@gmail.com
}

lous scaling behaviour of cuprates. Perhaps that triggers further explorations of the non-linear electrodynamic models. A string inspired Dirac-Born-Infeld (DBI) model was analysed in [29] to obtain DC conductivity along the method purposed in [30,31]. This was further generalised in [32] and [33] leading to linear scaling of resistivity with a temperature. More general non-linear electrodynamics was considered [34,35].

Considering the success of non-linear electrodynamic models it is natural to study the Fermi surface and excitations around it. On that score, [36] has studied the fermionic behaviour for string inspired DBI model. In the present work, we will consider another non-linear model of electrodynamics known as arcsin electrodynamics, which was introduced in [37]. An attractive feature of this model is the finite electric field of a pointlike charge at the origin and static electric energy of a particle is also finite [38,39]. The dual boundary theory of this model has been analysed in several works. By introducing a scalar field in the gravity theory, it was shown [39], that the boundary theory may undergo condensation, that corresponds to spontaneous breaking of a $U(1)$ symmetry and thus it admits a superconducting phase [39]. This model was further extended by introducing a neutral scalar field and axions, where the backreaction on the metric was also considered. This extended model exhibits metal/insulator transition with a variation of certain parameter of the theory, showing additional phase structure. Thus among the possible non-linear electrodynamics, it has been established that this particular theory has a wide spectrum of phases. In particular, as has been shown in [35], this model shows a linear temperature dependence of resistivity in a probe limit and thus it can capture the properties of direct conductivity of cuprates in the strange metal phase. However, analysis of Fermi surface and excitations around it has not been done yet and the aim of the present work is to fill in this gap. For this model, we have studied the nature of fermionic behaviour over the range of several parameters. We find there is a transition/crossover from Fermi liquid to non-Fermi liquid phase occurring due to variation of charge 
density of the system. We have also studied the fermionic excitations in this probe limit as well, which may give some insight on the underlying mechanism for such anomalous behaviour of cuprates.

The plan of the article is as follows. In the next Sect. 2, we will briefly introduce the nonlinear arcsin electrodynamics model and its blackhole solution. We will also discuss the probe limit and its background solution. In Sect. 3, we introduce Fermions and compute the Green's function of the dual operator. In Sect. 4, we present our results where we numerically solve the Dirac equation to obtain the Green's function and study its nature with the variation of different parameters. In Sect. 5 we conclude with a discussion.

\section{Bosonic part}

In this section, we revisit the model of non-linear arcsin electrodynamics which is interacting with gravity introduced in [35]. The action is given by

$$
\begin{aligned}
S= & \int d^{4} x \sqrt{-g}\left[R-\frac{1}{2}(\partial \phi)^{2}-V(\phi)-\frac{Y}{2} \sum_{I=1}^{2} \partial\left(\psi^{I}\right)^{2}\right] \\
& +S_{0},
\end{aligned}
$$

with

$$
S_{0}=\int d^{4} x \sqrt{-g}\left[-Z_{1}(\phi) \sin ^{-1}\left(Z_{2}(\phi) \mathcal{F}\right)\right]
$$

where $\psi^{(I)}$ are the two axions and $\phi$ is the scalar field whose potential is given by $V(\phi) . S_{0}$ is the action for non-linear electrodynamics where $\mathcal{F}=\frac{1}{4} F_{\mu \nu} F^{\mu \nu} . Z_{1}(\phi)$ and $Z_{2}(\phi)$ are the coupling constant which depends on the scalar $\phi$. One may observe that the Eq. (2.2) reduces to the usual Maxwell action at the limit of vanishing gauge field $A_{\mu} \rightarrow 0$ with the choice of $Z_{2}=1$ and $Z_{1}=1$.

The equation of motion for the above action turns out to be as follows. From the Einstein part of the action we have

$$
\begin{aligned}
R_{\mu \nu}= & \frac{1}{2}\left(\partial_{\mu} \phi\right)\left(\partial_{\nu} \phi\right) \\
& +\frac{Y}{2}\left(\partial_{\mu} \psi^{I}\right)\left(\partial_{\nu} \psi^{I}\right)+\frac{1}{2} g_{\mu \nu} V+\theta_{\mu \nu},
\end{aligned}
$$

where $\theta_{\mu \nu}$ can be expressed in terms of energy momentum tensor of gauge fields $T_{\mu \nu}$ as $\Theta_{\mu \nu}=T_{\mu \nu}-\frac{1}{2} g_{\mu \nu} T_{\lambda}^{\lambda}$ and in the second term on right hand side sum over $I$ is implied.

The equation for scalar and axion fields $\phi$ and $\psi^{I}$ are given by

$$
\begin{aligned}
& \nabla_{\mu} \nabla^{\mu} \phi-V^{\prime}(\phi) \\
& -\frac{Y^{\prime}}{2}\left(\partial \psi^{I}\right)^{2}-Z_{1}^{\prime}(\phi) \sin ^{-1}\left(Z_{2} \mathcal{F}\right)
\end{aligned}
$$

$$
\begin{aligned}
& -Z_{1}(\phi) Z_{2}^{\prime}(\phi) \frac{\mathcal{F}}{\sqrt{\left.1-\left(Z_{2}(\phi) \mathcal{F}\right)^{2}\right)}}=0, \\
& \nabla_{\mu}\left(Y \nabla^{\mu} \psi^{I}\right)=0 .
\end{aligned}
$$

The equation for gauge field turns out to be

$\nabla_{\mu}\left(\frac{Z_{1}(\phi) Z_{2}(\phi) \sqrt{-g}}{\sqrt{1-\left(Z_{2}(\phi) \mathcal{F}\right)^{2}}} F^{\mu \nu}\right)=0$.

The quantity $\theta_{\mu \nu}$, which is related to the energy-momentum tensor of the gauge field is given by

$$
\begin{aligned}
\theta_{\mu \nu}= & \frac{1}{2}\left[\frac{Z_{1}(\phi) Z_{2}(\phi) \sqrt{-g}}{\sqrt{1-\left(Z_{2}(\phi) \mathcal{F}\right)^{2}}}\left(F_{\mu \alpha} F_{\nu}{ }^{\alpha}-\frac{1}{4} g_{\mu \nu} F_{\alpha \beta} F^{\alpha \beta}\right)\right. \\
& \left.+Z_{1}(\phi) g_{\mu \nu} \sin ^{-1}\left(Z_{2}(\phi) \mathcal{F}\right)\right] .
\end{aligned}
$$

The expression of the current $J^{\mu}$ in the dual field theory is

$J^{\mu}=-\frac{\sqrt{-g} Z_{1}(\phi) Z_{2}(\phi)}{\sqrt{1-\left(Z_{2}(\phi) \mathcal{F}\right)^{2}}} F^{r \mu}$,

which is to be evaluated at the boundary.

In order to get the black hole solution, we will consider the following ansatz for the metric, gauge field, scalar field and axions

$$
\begin{aligned}
d s^{2} & =-D(r) d t^{2}+B(r) d r^{2}+C(r)\left(d x^{2}+d y^{2}\right), \\
\phi & =\phi(r), \\
A & =A_{t}(r) d t+\frac{h}{2}(x d y-y d x), \quad \psi^{1}=k_{p} x, \\
\psi^{2} & =k_{p} y,
\end{aligned}
$$

where $h$ is the magnetic field which lies on $x-y$ plane. The scalar field $\phi$ only depends on radial direction $r$. The axions $\psi^{I}$ is set up in $x$ and $y$ direction with the magnitude of momentum dissipation $k_{p}$ which breaks the translational symmetry at the boundary. Since we are only interested on the electrically charged solution of the black hole, we are setting $h$ to be zero.

The equations are quite involved, so we will consider the particular family of the solution by following the ansatz given in $[32,35]$

$$
\begin{aligned}
Z_{1}(\phi) & =z_{1}, \quad Z_{2}(\phi)=Y=1, \\
V & =-V_{0}, \quad \phi(r)=0, \quad B(r) D(r)=1,
\end{aligned}
$$

$z_{1}$ and $V_{0}$ are constants where we consider $z_{1}=1$ for further simplification. After taking these assumptions, the above equation of motion can be solved exactly. The solution for the metric coefficients are given in [35], where $C(r)$ is solved exactly and is given by

$C(r)=r^{2}$. 
Substituting $C(r)$ in the above equation of motion for scalar, the equation for the metric coefficient $D(r)$ becomes

$$
\begin{aligned}
r D^{\prime} & +D+\frac{k_{p}^{2}}{2}-\frac{1}{2} r^{2} V_{0} \\
& +\frac{r^{2}}{2}\left[-\frac{\mathcal{F}}{\sqrt{1-\mathcal{F}^{2}}}+\sin ^{-1} \mathcal{F}\right]=0 .
\end{aligned}
$$

Solving the above first order differential equation and the equation of motion for the gauge field the coefficients $D(r)$ of the metric and the gauge field are given by

$$
\begin{aligned}
D(r)= & \frac{1}{36 r^{2}}\left[-18 k_{p}^{2} r^{2}+6 V_{0} r^{4}\right. \\
& -7 \sqrt{2} r^{2} \sqrt{-r^{4}+\sqrt{r^{8}+\rho^{4}}} \\
& -6 r^{4} \sin ^{-1} \frac{r^{4}-\sqrt{r^{8}+\rho^{4}}}{\rho^{2}} \\
& -36 M r+42^{1 / 4} \rho r\left(-r^{4}\right. \\
& \left.+\sqrt{r^{8}+\rho^{4}}\right)^{1 / 4}{ }_{2} F_{1}(1 / 8,3 / 4,9 / 8 ; \\
& \frac{\left.\left.2 r^{8}+\rho^{4}-2 r^{4} \sqrt{r^{8}+\rho^{4}}\right)\right],}{\rho^{4}} \\
A_{t}(r)= & \int_{r_{h}}^{r} \sqrt{-2 \mathcal{F}} d r \quad \text { with } \quad \mathcal{F}=\frac{r^{4}-\sqrt{r^{8}+\rho^{4}}}{\rho^{2}},
\end{aligned}
$$

where $\rho$ is the charge density of the system and $M$ mass of the blackhole. $M$ is fixed by $D(r)$ which vanishes at the horizon $\left(D\left(r=r_{h}\right)=0\right)$. The temperature of the blackhole is given by

$T=\frac{1}{8 \pi r_{h}^{3}}\left(\mathcal{F} \Omega r_{h}^{4}-2 \sin ^{-1}(\mathcal{F}) r_{h}^{2}-k_{p}^{2} r_{h}^{2}-V_{0} r_{h}^{4}\right)$,

where we have defined $\Omega=\frac{1}{\sqrt{1-\mathcal{F}^{2}}}$. In the expression of temperature, $\mathcal{F}$ is evaluated at horizon $r=r_{h}$.

\subsection{Probe geometry}

As has been pointed out that strong momentum dissipation limit may be considered as a probe limit [35], where one can ignore the backreaction on the metric. In this probe limit we will consider hyperscaling violating geometry as the background, which may reproduce linear temperature dependence of resistivity [34]. The hyperscaling geometry appears as a solution [34] with the coupling given by $V(\phi) \sim-V_{0} e^{\eta \phi}$ and $Y(\phi) \sim e^{\alpha \phi}$, where $\alpha$ and $\eta$ are two constants. The ansatz for the metric and other fields for hyperscaling violating geometry are given by

$$
\begin{aligned}
d s^{2} & =r^{-\theta}\left(-f(r) r^{2 z} d t^{2}+\frac{L^{2} d r^{2}}{r^{2} f(r)}+r^{2}\left(d x^{2}+d y^{2}\right)\right), \\
\phi & =\kappa \ln (1 / r), \quad \psi^{1}=k_{p} x, \quad \psi^{2}=k_{p} y, \quad
\end{aligned}
$$

where $\kappa, k_{p}$ and $L$ are constants. $z$ and $\theta$ are the Lifshitz scaling and hyperscaling violation exponents respectively. The solution with given ansatz has been studied in [35], where the metric coefficient $f(r)$ and other parameters are

$$
\begin{aligned}
f(r) & =1-\left(\frac{r_{0}}{r}\right)^{2+z-\theta}, \quad z=\frac{\alpha^{2}-\eta^{2}+1}{\alpha(\alpha-\eta)}, \quad \theta=\frac{2 \eta}{\alpha}, \\
\kappa & =-\frac{2}{\alpha}, \quad L^{2}=\frac{(z+2-\theta)(2 z-\theta)}{V_{0}}, \\
k^{2} & =\frac{2 V_{0}(z-1)}{2 z-\theta} .
\end{aligned}
$$

Here, $r_{h}$ is the radius of horizon satisfying $f\left(r_{h}\right)=0$. The gauge field can be easily obtained by solving the equation of motion given in [26]. Here, we will only give the expression for the gauge field for this background which turns out to be

$$
\begin{aligned}
A_{t} & =\int_{r_{h}}^{r} r^{z-\theta-1} \sqrt{-2 \tilde{\mathcal{F}}} d r, \text { with } \\
\tilde{\mathcal{F}} & =\frac{\tilde{C}-\sqrt{\tilde{C}^{2}+Z_{2}^{2} \rho^{4}}}{Z_{2}^{2} \rho^{2}}
\end{aligned}
$$

where we have defined $\tilde{C}=\left(Z_{1} Z_{2} r^{2(1-\theta)}\right)^{2}$ with $Z_{1} \sim e^{\gamma \phi}$ and $Z_{2} \sim e^{\delta \phi}$. The temperature is given by

$T=\frac{(2+z-\theta)}{4 \pi L} r_{h}^{z}$.

We would like to comment on the parameters $z$ and $\theta$ before proceeding. $z=1$ and $\theta=0$ corresponds to AdS background. To have a well-defined geometry and resolvable singularity, the range of these two parameters is restricted. This restriction arises by considering the Gubser criterion in conjunction with the null energy condition

$$
\begin{aligned}
& \operatorname{IR} r \rightarrow \infty:(1<z<2, \quad \theta<2 z-2), \quad(z>2, \quad \theta<2), \\
& \operatorname{IR} r \rightarrow 0:(0<z<2, \quad \theta>z+2), \quad(z \leq 2, \quad \theta>2) .
\end{aligned}
$$

In addition to this, in order to have linear temperature dependence of resistivity of cuprates one need to satisfy

$\frac{\gamma}{\alpha}=\frac{\lambda-2}{2} z+2, \quad \frac{\delta}{\alpha}=-\frac{\lambda-2}{2} z-2, \quad \theta=4-2 z$.

provided $\lambda \geq 0$, for simplicity we will be taking $\lambda=0$. Even though this behaviour arises in high temperature limit, one can choose the appropriate value of the parameters to retain the behaviour of cuprate at sufficiently low temperature. 


\subsection{Near Horizon limit}

The nature of the fermionic excitation is closely related to the near horizon structure of the metric. In this regard, we will analyse near horizon structure of the metric given in Eqs. (2.8) and (2.14). To study the fermionic spectral function we will consider two different backgrounds; First, we will consider fully backreacted background which is given in (2.8) and second we will consider the hyperscaling violating geometry which arises in the probe limit. We will begin with the discussion of the former with extremal case $(T=0)$.

\subsubsection{Backreacted geometry}

The full metric for fully backreacted geometry is given in (2.8), we would like to rewrite this metric in the following form

$d s^{2}=-r^{2} f(r) d t^{2}+\frac{d r^{2}}{r^{2} f(r)}+r^{2} d \vec{x}^{2}$,

where $f(r)=D(r) / r^{2}$. The horizon is defined by a point $r_{h}$ where $D\left(r_{h}\right)=0$ or $f\left(r_{h}\right)$ vanishes, similarly we will define a point $r_{*}$ such that the derivative of $f(r)$ at $r_{*}$ vanishes $\left(f^{\prime}\left(r_{*}\right)=0\right)$. However, in the limit of zero temperature, these two points coincide with each other i.e. $r_{*}=r_{h}$.

First, we begin the near horizon expansion of the metric in this limit of zero temperature. The metric coefficient $f(r)$ at the near horizon $\left(r \rightarrow r_{h}\right)$ develops a double zero which is given by

$$
\begin{aligned}
f(r) & =\frac{\left(r-r_{h}\right)^{2}}{L_{2}^{2}}, \\
L_{2} & =\frac{1}{r_{h}} \sqrt{\frac{2}{V_{0}-\sin ^{-1}(\mathcal{F})+\left(\Omega-\frac{1}{2} \partial_{r} \Omega\right) \mathcal{F}}},
\end{aligned}
$$

where all the quantities in $L_{2}$ are evaluated at $\left(r=r_{h}\right)$. A natural scaling factor $L_{2}$ of the metric occurs in the near horizon limit. We have used Eq. (2.11) to find $D(r)$ and $L_{2}$. Following [16] we will consider the scaling limit

$r-r_{*}=\epsilon \frac{L_{2}^{2}}{\zeta}, \quad t=\epsilon^{-1} \tau, \quad \epsilon \rightarrow 0 \quad$ with $\tau, \zeta$ finite.

In this limit the metric (2.8) and the gauge field (2.12) are given by

$d s^{2}=\frac{L_{2}^{2}}{\zeta^{2}}\left(-d \tau^{2}+d \zeta^{2}\right)+\frac{r_{h}^{2}}{L_{2}^{2}} d \overrightarrow{x^{2}}, \quad A_{\tau}=\frac{e}{\zeta}$,

where $e=\sqrt{-2 \mathcal{F}} L_{2}^{2}$ evaluated at horizon $\left(r=r_{h}\right)$. We can see from the above Eq. (2.24), that the structure of the metric (2.8) at near horizon becomes $A d S_{2} \times \mathbb{R}^{2}$, with $L_{2}$ being the radius of curvature of $A d S_{2}$.
To generalise this in the case of finite temperature, where $r_{*} \neq r_{h}$ one considers the following equation in addition to (2.23) [16,22]

$r_{h}-r_{*}=\epsilon \frac{L_{2}^{2}}{\zeta_{0}}$ with $\zeta_{0}$ finite,

the metric under the additional equation turns out to be blackhole in $A d S_{2} \times \mathbb{R}^{2}$ given by

$d s^{2}=\frac{L_{2}}{\zeta^{2}}\left(-\left(1-\frac{\zeta^{2}}{\zeta_{0}^{2}}\right) d \tau^{2}+\frac{\zeta^{2}}{1-\frac{d \zeta^{2}}{\zeta_{0}^{2}}}\right)+\frac{r_{*}^{2}}{L_{2}^{2}} d \overrightarrow{x^{2}}, \quad$ with

$A_{\tau}=\frac{e}{\zeta}\left(1-\frac{\zeta}{\zeta 0}\right)$

where the temperature is defined as $T=\frac{1}{2 \pi \zeta_{0}}$.

It may be interesting to check the asymptotic limit of this geometry which turns out to be $A d S_{4}$ given by

$$
\begin{aligned}
& d s_{4}^{2}=r^{2}\left(-f(r) d t^{2}+d \vec{x}^{2}\right)+\frac{d r^{2}}{r^{2} f(r)}, \\
& f(r)=1-\frac{k_{p}^{2}}{2 r^{2}}+\frac{b_{1}}{r^{3}},
\end{aligned}
$$

where we have defined $b_{1}$ as

$$
\begin{aligned}
b_{1}= & -1+\frac{1}{2} k_{p}^{2}+\frac{7}{18 \sqrt{2}} c_{1}-\frac{1}{6} \sin ^{-1}\left(\frac{c_{1}}{\rho}\right)^{2} \\
& -\frac{\left(2 c_{1}^{2}\right)^{\frac{1}{4}} \rho}{9}{ }_{2} F_{1}\left(\frac{1}{8}, \frac{3}{4} ; \frac{9}{8} ; 1-\frac{2 c_{1}^{2}}{\rho^{4}}\right),
\end{aligned}
$$

with $c_{1}^{2}=-1+\sqrt{1+\rho^{4}}$.

\subsubsection{Probe geometry}

In the hyperscaling violating geometry, one can consider the similar near horizon expansion $\left(r \rightarrow r_{h}\right)$. Before proceeding, we will consider the following general coordinate transformation $r=u^{\frac{1}{z-\theta}}$, under which the metric (2.14) turns out to be

$$
\begin{aligned}
d s^{2} & =-u^{2} F(u) d t^{2}+\frac{\hat{L}^{2}}{u^{2} F(u)} d u^{2}+u^{\frac{2-\theta}{z-\theta}} d \vec{x}^{2}, \\
F(u) & =u^{\frac{\theta}{z-\theta}}\left(1-\left(\frac{u_{h}}{u}\right)^{\frac{2+z-\theta}{z-\theta}}\right),
\end{aligned}
$$

where $u_{h}$ is defined as $F\left(u_{h}\right)=0$ and $\hat{L}=L /(z-\theta)$.

In the extremal limit, the metric at near horizon turns out to be $A d S_{2} \times \mathbb{R}^{2}$ with the metric and gauge field given by

$$
\begin{aligned}
d s^{2} & =\frac{L_{2}}{\zeta^{2}}\left(-d \tau^{2}+d \zeta^{2}\right)+u_{h}^{\frac{2-\theta}{z-\theta}} d \vec{x}^{2}, \\
A_{\tau} & =\frac{e}{\zeta}, \quad L_{2}^{2}=\frac{2(z-\theta)^{2}}{a_{1}^{2} u_{h}^{2}} \hat{L}^{2},
\end{aligned}
$$


where $e=\sqrt{-2 \tilde{\mathcal{F}}}$ and $a_{1}=F^{\prime \prime}(u)$ are both evaluated at $u_{*} \cdot{ }^{1}$ To deduce the above near horizon metric, we have chosen the following scaling limits

$u-u_{*}=\epsilon \frac{L_{2}^{2}}{\zeta}, \quad t=\frac{L_{2}}{L} \epsilon^{-1} \tau, \quad \epsilon \rightarrow 0 \quad$ with $\tau, \zeta$ finite.

In the finite temperature, one can consider the additional scaling limit given in (2.25) and follow the similar procedure. On doing so, the metric turns out to be a blackhole in $A d S_{2} \times \mathbb{R}^{2}$, with metric and the gauge field given by

$$
\begin{aligned}
d s^{2} & =\frac{L_{2}}{\zeta^{2}}\left(-\left(1-\frac{\zeta^{2}}{\zeta_{0}^{2}}\right) d \tau^{2}+\frac{d \zeta^{2}}{1-\frac{\zeta^{2}}{\zeta_{0}^{2}}}\right)+u_{*}^{\frac{2-\theta}{z-\theta}} d \vec{x}^{2} \\
A_{\tau} & =\frac{e}{\zeta}\left(1-\frac{\zeta}{\zeta_{0}}\right),
\end{aligned}
$$

where $L_{2}$ and $e$ are given in (2.30).

\section{Green's function}

In order to probe the system, we will consider Dirac action in fully backreacted geometry and hyperscaling violating geometry as well. The action is given by

$$
\begin{aligned}
S= & i \int d^{4} x \sqrt{-g} \bar{\psi}\left(\Gamma^{a} D_{a}-m\right. \\
& \left.-\frac{1}{2} i p \Gamma^{\mu \nu}\left(e_{\mu}\right)^{a}\left(e_{\nu}\right)^{b} F_{a b}\right) \psi,
\end{aligned}
$$

where the first term corresponds to the kinetic term, the second term is the mass of the spin $1 / 2$ particle and the last term corresponds to the coupling of Dirac field with the electrodynamic field, where the strength of the coupling is given by the dipole coupling $p$. The covariant derivative and Gamma matrices are given by,

$D_{a}=\partial_{a}+\frac{1}{4}\left(\omega_{\mu \nu}\right)_{a} \Gamma^{\mu v}-i q A_{a}$,

$\Gamma^{a}=e_{\mu}^{a} \Gamma^{\mu}, \quad \Gamma^{\mu \nu}=\frac{1}{2}\left[\Gamma^{\mu}, \Gamma^{\nu}\right]$,

$\left(e_{\mu}\right)^{a}$ is the vielbein, $\omega_{\mu \nu}$ is the spin connection and $q$ is the charge of the spin $1 / 2$ particle. The equation of motion for the above action is given by,

$\left(\Gamma^{a} D_{a}-m-\frac{1}{2} i p \Gamma^{\mu \nu}\left(e_{\mu}\right)^{a}\left(e_{\nu}\right)^{b} F_{a b}\right) \psi=0$.

To eliminate the spin connection in the Dirac Eq. (3.3), we will consider the following ansatz $\psi=\left(-g g^{r r}\right)^{\frac{1}{4}}$ $e^{-i \omega t+i k_{i} x^{i}} \lambda(r)$. For further simplification, we will now introduce the projection operator $[24,26] \Gamma_{ \pm}=\frac{1}{2}\left(1 \pm \Gamma^{r} \Gamma^{t} \hat{k} . \vec{\Gamma}\right)$

\footnotetext{
${ }^{1} u_{*}=u_{h}$ in the case of extremal limit $(T=0)$.
}

and rewrite $\lambda(r)$ as $\lambda_{ \pm}=\Gamma_{ \pm} \lambda(r)$, where $\hat{k}$ is the unit vector along the spatial direction. Our choices of Gamma matrices are

$$
\begin{aligned}
\Gamma^{t}=\left(\begin{array}{cc}
i \sigma_{1} & 0 \\
0 & i \sigma_{1}
\end{array}\right), & \Gamma^{r}=\left(\begin{array}{cc}
-\sigma_{3} & 0 \\
0 & -\sigma_{3}
\end{array}\right), \\
\Gamma^{x}=\left(\begin{array}{cc}
-\sigma_{2} & 0 \\
0 & \sigma_{2}
\end{array}\right), & \Gamma^{y}=\left(\begin{array}{cc}
0 & -i \sigma_{2} \\
i \sigma_{2} & 0
\end{array}\right) .
\end{aligned}
$$

We can write $\lambda_{ \pm}$in terms of two component spinor given by $\lambda_{ \pm}=\left(\begin{array}{l}\lambda_{1 \pm} \\ \lambda_{2 \pm}\end{array}\right)$. In addition, we use the spatial rotational symmetry to set $k_{i}=k$ for $i=x$ and zero for $i=y$. With the above choices together with Gamma matrices the Dirac equation reduces to

$$
\begin{aligned}
\left(\partial_{r}\right. & \left.+m \sqrt{g_{r r}}\right) \lambda_{1 \pm} \\
& =\left(\sqrt{\frac{g_{r r}}{g_{t t}}}\left(\omega+q A_{t}\right) \pm \sqrt{\frac{g_{r r}}{g_{x x}}} k+p \sqrt{g_{t t}} \partial_{r} A_{t}\right) \lambda_{2 \pm}, \\
\left(\partial_{r}\right. & \left.-m \sqrt{g_{r r}}\right) \lambda_{2 \pm} \\
& =\left(-\sqrt{\frac{g_{r r}}{g_{t t}}}\left(\omega+q A_{t}\right) \pm \sqrt{\frac{g_{r r}}{g_{x x}}} k+p \sqrt{g_{t t}} \partial_{r} A_{t}\right) \lambda_{1 \pm},
\end{aligned}
$$

Before discussing solution of this Dirac Eq. (3.5) let us consider the near horizon limit of it. We will consider only the backreacted geometry for this purpose. For the extremal case $(T=0)$, using (2.24), Dirac equation simplifies into

$$
\begin{aligned}
\zeta \partial_{\zeta}\left(\begin{array}{l}
\lambda_{1 \pm} \\
\lambda_{2 \pm}
\end{array}\right) & =U\left(\begin{array}{c}
\lambda_{1 \pm} \\
\lambda_{2 \pm}
\end{array}\right), \\
U & =\left(\begin{array}{cc}
-m L_{2} & q e+\frac{k}{r_{h}} L_{2}+\frac{p e}{L_{2}} \\
-q e+\frac{k}{r_{h}} L_{2}+\frac{p e}{L_{2}} & m L_{2}
\end{array}\right) .
\end{aligned}
$$

The solution to this equation is given by $\lambda_{ \pm}=a E_{+} \zeta^{-v}+$ $b E_{-} \zeta^{\nu}$, where $E_{ \pm}$are the real eigen vector of $U$ and the exponent is the eigen value of $U$ given by

$$
v=\sqrt{\left(m^{2}+\left(\frac{k}{r_{h}}+\frac{p e}{L_{2}}\right)^{2}\right) L_{2}^{2}-q^{2} e^{2}} \quad(T=0) .
$$

For the finite temperature, calculation remains similar except one has to consider the near horizon metric and gauge field for the finite temperature given in Eq. (2.26). In this case, the eigenvalue $v$ is given by

$$
v=\sqrt{\left(m^{2}+\left(\frac{k}{r_{*}}+\frac{p e}{L_{2}}\right)^{2}\right) L_{2}^{2}-q^{2} e^{2}} \quad(T>0) .
$$


$v$ plays a central role in determining the nature of the Fermi surface. The lifetime of the fermionic excitations around the Fermi surface is given by [22] $\tau=\omega^{-v}$ at zero temperature and $\tau \sim T^{-2 v}$ at finite temperature. As the lifetime of the excitation depends on $v$, the metallic behaviour of the system is controlled by $v$.

In the region of phase diagram with $v \geq 1$, we have a normal metal phase, where the excitation of the quasiparticles is governed by Landau Fermi liquid. However, in the region where $1 / 2<v<1$ we get stable quasiparticles, whose lifetime scales differently from that of Landau Fermi liquid theory. In the region with $v<1 / 2$, we have a short lived quasiparticles which are the characteristic of strange metal phase, this region is called non-Fermi liquid region. In between the regime of stable and non-stable quasiparticle, there is a transition point where $v=1 / 2$, this region corresponds to a marginal Fermi liquids regime. The conformal dimension of the dual operator in the IR CFT will be $\Delta=v+\frac{1}{2}$. There is a range of momentum $k$, known as oscillatory region $[24,26]$, for which $v$ becomes complex thus leading to complex dimension of the dual operator.

Coming back to the solution of the Dirac Eq. (3.5) for the full metric, we need a boundary condition. For that purpose we define $\eta \pm=\frac{\lambda_{1 \pm}}{\lambda_{2}}$, which yield the flow equation given by

$$
\begin{aligned}
& \left(\partial_{r}+2 m \sqrt{g_{r r}}\right) \eta_{ \pm}-\left(v_{-} \pm k \sqrt{\frac{g_{r r}}{g_{x x}}}\right) \\
& -\left(v_{+} \mp \sqrt{k \frac{g_{r r}}{g_{x x}}}\right) \eta_{ \pm}^{2}=0,
\end{aligned}
$$

where

$v_{ \pm}=\sqrt{\frac{g_{r r}}{g_{x x}}}\left(\omega+q A_{t}\right) \pm p \partial_{r} A_{t}$.

To solve the above Eq. (3.9) numerically, we impose the infalling boundary condition given by

$\eta \pm=i$ for $r \rightarrow r_{h}$

The boundary retarded Green's function is given by

$G_{R}=\lim _{r \rightarrow \infty} r^{2 m}\left(\begin{array}{cc}\eta_{+} & 0 \\ 0 & \eta_{-}\end{array}\right)$.

In the background with hyperscaling violating geometry in the UV limit, the boundary Green's function in the regime of linear response has been derived in [40] and is given by

$G_{R}=\lim _{r \rightarrow \infty}\left(\begin{array}{cc}\eta_{+} & 0 \\ 0 & \eta_{-}\end{array}\right)$

Comparing (3.12) and (3.13), one may notice that for the massless case, the two Green's function matches. We can see from the Eq. (3.9) that the two diagonal part of the retarded Green function are related to each other by flipping the sign of $k$ i.e. $G_{11}(\omega, k)=G_{22}(\omega,-k)$. So it is sufficient to evaluate the Green's function for only one from the two, hence we will be only considering $G_{22}$ for our calculation.

In the next section, we will be studying the behaviour of the spectral function given by $A(\omega, k)=\operatorname{Im}\left(G_{22}\right)$ with respect to variation in charge density $\rho$, charge of the particle $q$ and the momentum dissipation term $k_{p}$ in zero and finite temperature for fully backreacted background. In case of the hyperscaling violating geometry, we will study the fermionic spectrum for a specific choice of background parameter which leads to linear temperature response of resistivity.

\section{Result}

In this section, we consider the behaviour of the Fermi surface associated with the operators dual to the fermionic modes. As mentioned earlier, we will limit ourselves to $G_{22}$. Considering $G_{22}$ means that we only consider $\eta_{-}$, which is sufficient, as the behaviour of the other Fermion for $\left(\eta_{+}\right)$will be similar i.e. if we find a Fermi surface for $\eta_{-}$at $k_{F}$ than the Fermi surface for $\eta_{+}$will be in $-k_{F}$. For the sake of simplicity, we will be considering the massless case with $m=0$. We will also set the value of the scalar potential to be $V_{0}=6$ and Pauli's coupling $p=0$. To begin, we numerically solve (3.9) where we impose the infalling boundary condition given in Eq. (3.11), and obtain the spectral function $A(\omega, k)$ as function of $\omega$ and $k$. In order to locate the Fermi surface, we will look for the poles of the Green's function in case of the zero temperature and in case of finite temperature we will follow $[24,28]$, where the position of Fermi surface is given by sharp peak in the plot of a spectral function around $k=k_{F}$ at $\omega=0$, which has a small enough width. In addition, if we plot the spectral function vs. $\omega$ at $k=k_{F}$ that should show a peak around $\omega=0$.

\subsection{Backreacted background}

In this subsection, we have studied the behaviour of the fermionic spectrum with variation of the parameters for the fully backreacted background.

From (2.13) fixing the value of the temperature $T$, we get a relation between the charge density $\rho$ and the momentum dissipation term $k_{p}$ and on choosing the value of one determines the value of the other. Here, the value of $k_{p}$ is determined by choosing the value of $\rho$ and vice versa.

First, we begin with the extremal case $T=0$, where we vary the charge density $\rho$ and look for the poles of the Green's function for two cases with charge $q=2$ and $q=1$. We have plotted $k_{F}$ versus the inverse of charge density $(1 / \rho)$ for $q=2$ in Fig. 1a and $q=1$ in Fig. 2a. Blue dots correspond to Fermi momenta $\left(k_{F}\right)$, which corresponds to poles of the Green's function. In the same figure, the shaded portion which is tapering toward the right represents an oscil- 


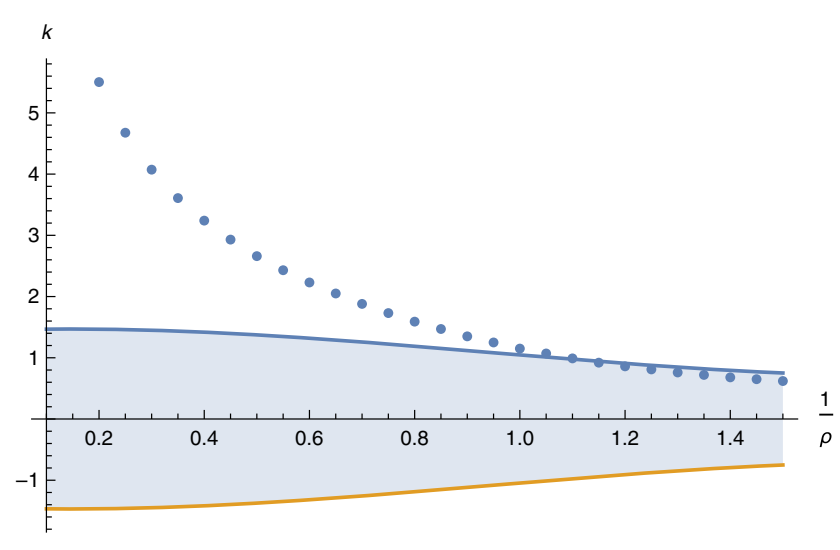

(a) $k$ vs. $\frac{1}{\rho}$ for $q=2$.

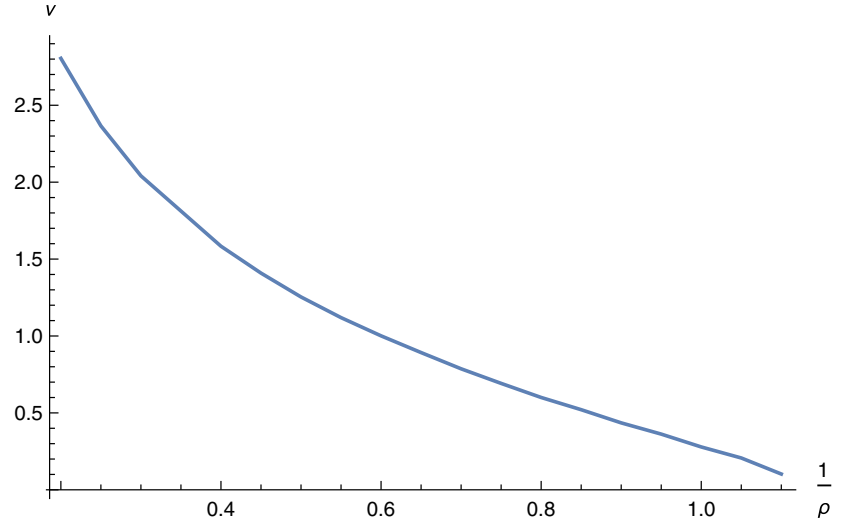

(b) $\nu$ vs. $\frac{1}{\rho}$ for $q=2$.

Fig. 1 The shaded region on the left figure corresponds to the oscillatory region, with blue dots representing the Fermi momenta $\left(k_{F}\right)$ for $q=2$. On right we have $v$ vs. $1 / \rho$ plot, where the quasiparticles start becoming stable with a higher value of charge density $\rho$

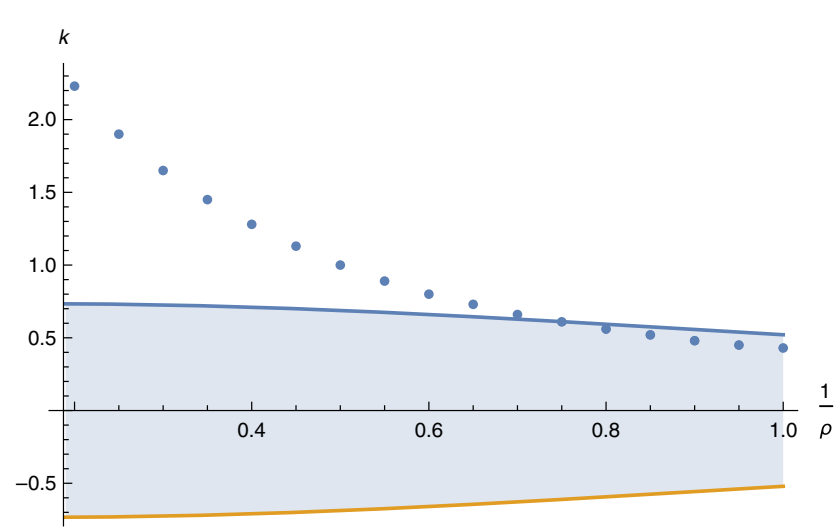

(a) $k$ vs. $\frac{1}{\rho}$ for $q=1$.

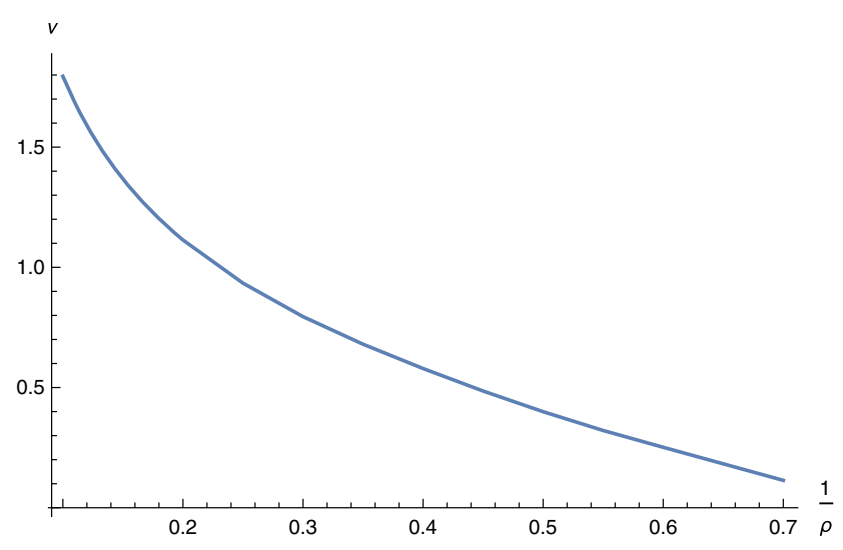

(b) $\nu$ vs. $\frac{1}{\rho}$ for $q=1$.

Fig. 2 The shaded region on the left figure corresponds to the oscillatory region, with blue dots representing the Fermi momenta $\left(k_{F}\right)$ for $q=1$. On right we have $\nu$ vs. $1 / \rho$ plot, where the quasiparticles start becoming stable with a higher value of charge density $\rho$

latory region with the two boundaries given by blue and brown lines. We can see from Fig. 1a as the charge density $(\rho)$ decreases the Fermi surface approaches the oscillatory region and enters around $\rho=0.8849$ where the Fermi surface ceases to exist. Similar result hold for the lower charge case $q=1$, which is given in Fig. 2a, with the decrease of charge density of the system Fermi surface, moves toward the oscillatory region and enters around $\rho=1.333$.

In order to study the change in nature of the fermionic excitations further, we have plotted $v$ versus inverse of charge density $(1 / \rho)$ of the system in Fig $1 \mathrm{~b}$ for $q=1$ and Fig $2 \mathrm{~b}$ for $q=2$. As we can observe that on decreasing the charge density $(\rho), v$ starts decreasing and vanishes around $\rho=0.8849$. Initially, at sufficiently large charge density, the system was in a Fermi regime, as one start decreasing the charge density the system starts to move from the regime of Fermi liquid enters the marginal Fermi regime at $\rho=1.1628$ where $v=1 / 2$ and reaches the non-Fermi regime. Further decrease of $\rho$ leads to vanishing of $v$ signalling non existence of the Fermi surface, where Fermi momenta are inside the oscillatory region. Thus with the decrease of charge density $\rho$, there is a transition from Fermi to the non-Fermi regime. This transition/crossover is taking place at zero temperature and may be related to a quantum phase transition. In addition, we can see from the comparison of the Figs. $1 b$ and $2 b$, that with the increase of charge of the particle $q$ the point of transition from Fermi to non-Fermi get shifted to the right of the parameter space.

In order to make the change of behaviour with momentum dissipation more explicit, we have plotted $v$ vs. $\sqrt{\rho} / k_{p}$ given in Fig. 3a, b for two different charges $q=1,2$, where we see on decreasing the momentum dissipation $k_{p}$ their is a transition from Fermi to non-Fermi regime. The red dashed line correspond to that marginal Fermi liquid regime with 


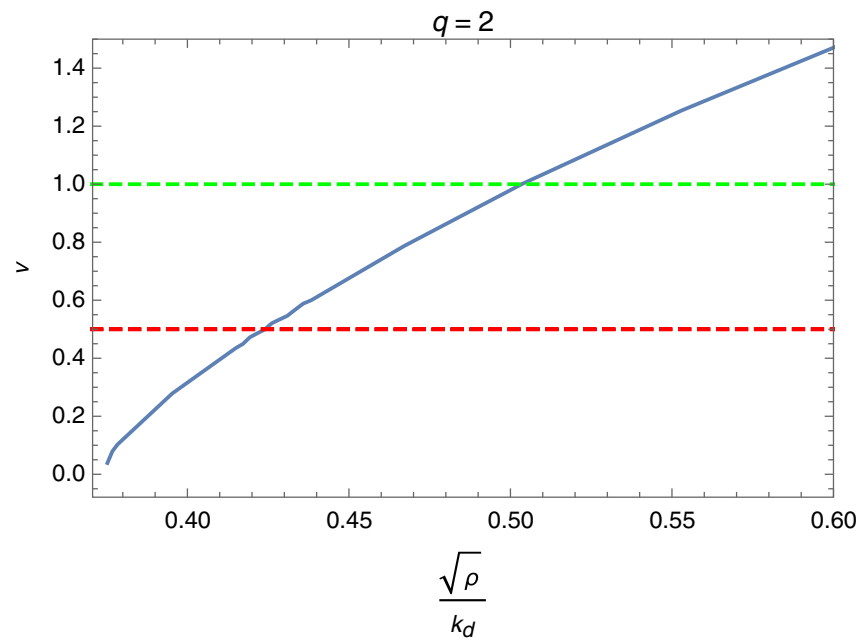

(a) $\nu$ vs. $\sqrt{\rho} / k_{p}$ for $q=2$.

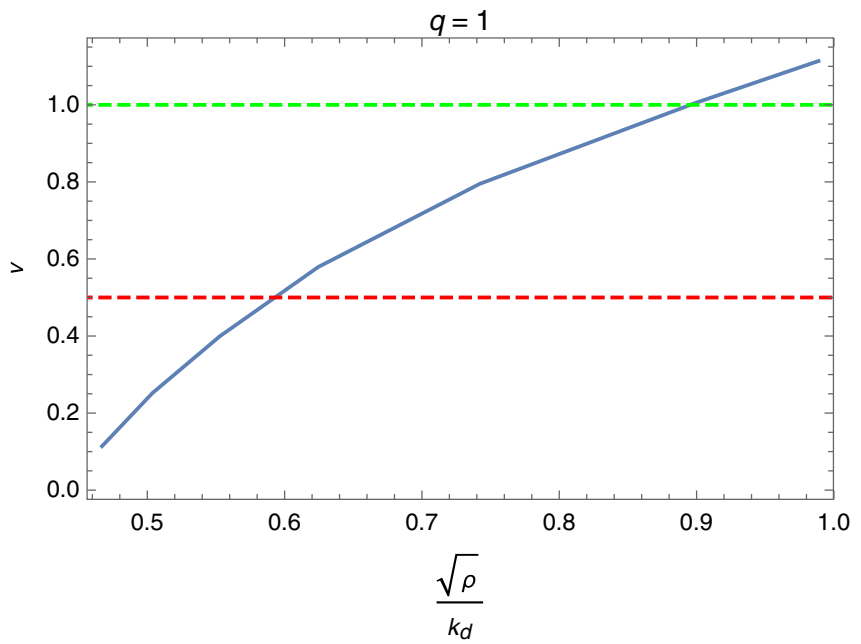

(b) $\nu$ vs. $\sqrt{\rho} / k_{p}$ for $q=1$.

Fig. $3 v$ vs. $\frac{\sqrt{\rho}}{k_{p}}$ plot. Red and green dashed line correspond to $v=1 / 2$ and $v=1$ line respectively

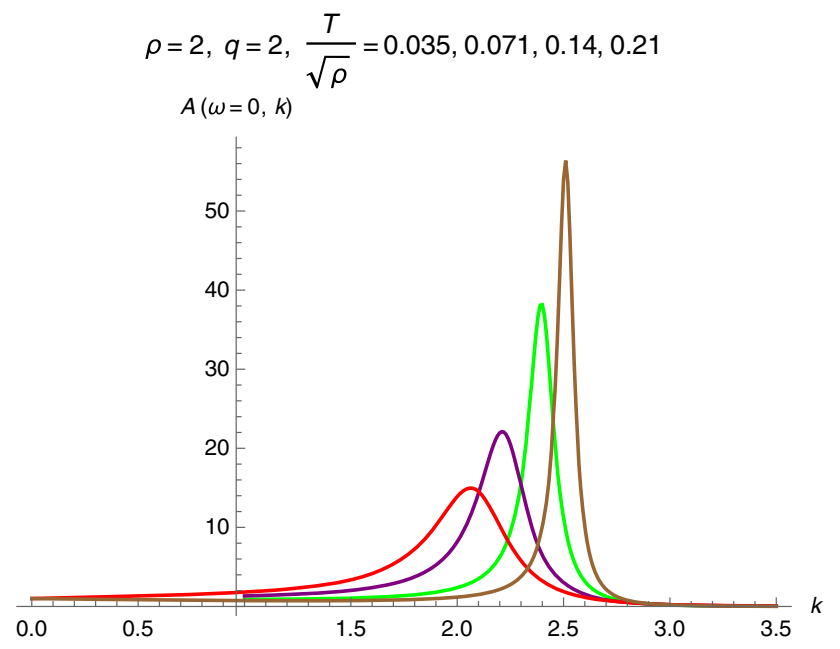

Fig. $4 A(\omega=0, k)$ vs. $k$ with $q, \rho=2$ for different values of temperature $T / \sqrt{\rho}=0.035$ (Brown), 0.075 (Green), 0.14 (Purple) and 0.21 (Red)

$v=1 / 2$, the upper plane above $v=1 / 2$ is the Fermi regime and lower plane correspond to the non-Fermi regime. There is a critical value of the momentum dissipation at which $v=$ $1 / 2$. As we move toward the upper plane, the quasiparticles become stable and finally enters the region of Landau Fermi liquid which is given by the region with and above the green dashed line in Fig. 3a, b. Comparing Fig. 3a, b we see for higher charge, $q=2$ the transition is occurring at a higher value of $k_{p}$. The transition is driven by the changing the magnitude $k_{p}$ of axionic scalars and can be thought of as a disorder driven transition [32].

After discussing the zero temperature case we will move to the finite temperature. We have plotted spectral function
$A(\omega=0, k)$ vs $k$ for four different temperatures starting from $T / \sqrt{\rho}=0.035$. We have set the value of charge density $\rho=$ 2 and $q=2$. We can see that with the increase in temperature the height of peak starts decreasing and becomes broader and move towards the left. With the increase in temperature, the density gets spread out due to thermal excitation leading to a broader peak (Fig. 4).

In order to see the change in the nature of fermionic excitations over the range of momentum dissipation with variation of temperature, we have given a density plot in $T / \sqrt{\rho}$ vs. $\sqrt{\rho} / k_{p}$ plane in Fig. $5 \mathrm{a}, \mathrm{b}$, with a colour gradient representing values of $v$, for two different values of charge $q=1$ and $q=2$. The value of $v$ increases from left to right, with darker and lighter shades corresponding to lower and higher values of $v$ respectively. The white region on the left is the oscillatory region and the green dashed line is the curve for $v=1 / 2$, where on the left of the curve we have strange metal phase and on the right, we have stable quasiparticle phase. The density plot extends for the higher value of $v>1$ which is given by the yellow dashed line representing a normal metal phase. We can see from the figures that, with an increase in temperature the critical value of momentum dissipation $k_{p}$ for a transition from the strange metal to the normal metal phase decreases. Even though for a given temperature the transition occurs on the specific value of momentum dissipation for different values of charge of the particle $q$, the qualitative behaviour does not depend on this variable $q$. This is similar to the normal metal to strange metal transition found in [22] where the transition occurred due to tuning of doping instead of a variation of the magnitude of axionic scalar.

In order to see the similar changes with charge density, in Fig. 6a we have a density plot in $T / \sqrt{\rho}$ vs. $\rho$ plane, where we can see the critical transition value of $\rho$ from strange 


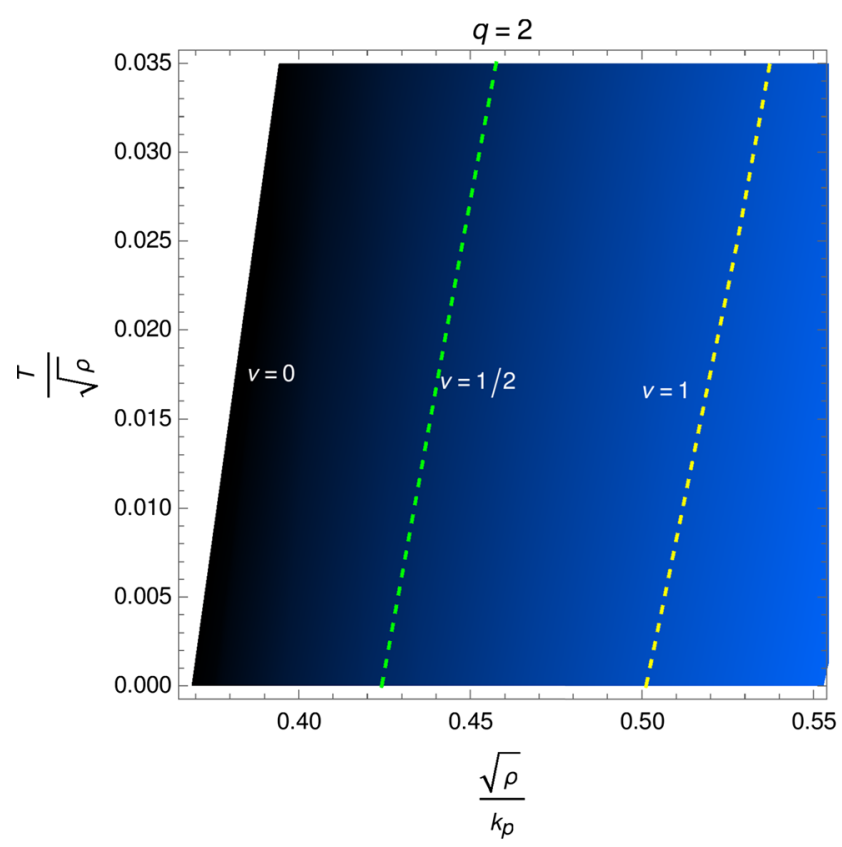

(a) $T / \sqrt{ } \rho$ vs. $\sqrt{\rho} / k_{p}$ plane for $q=2$.

Fig. 5 Density plot of $v$ in $T / \sqrt{\rho}$ vs. $\sqrt{\rho} / k_{p}$ plane. The colour gradient corresponds to the value of $v$ which increases from left to right. The white portion is the oscillatory region with darkest shades being $v=0$

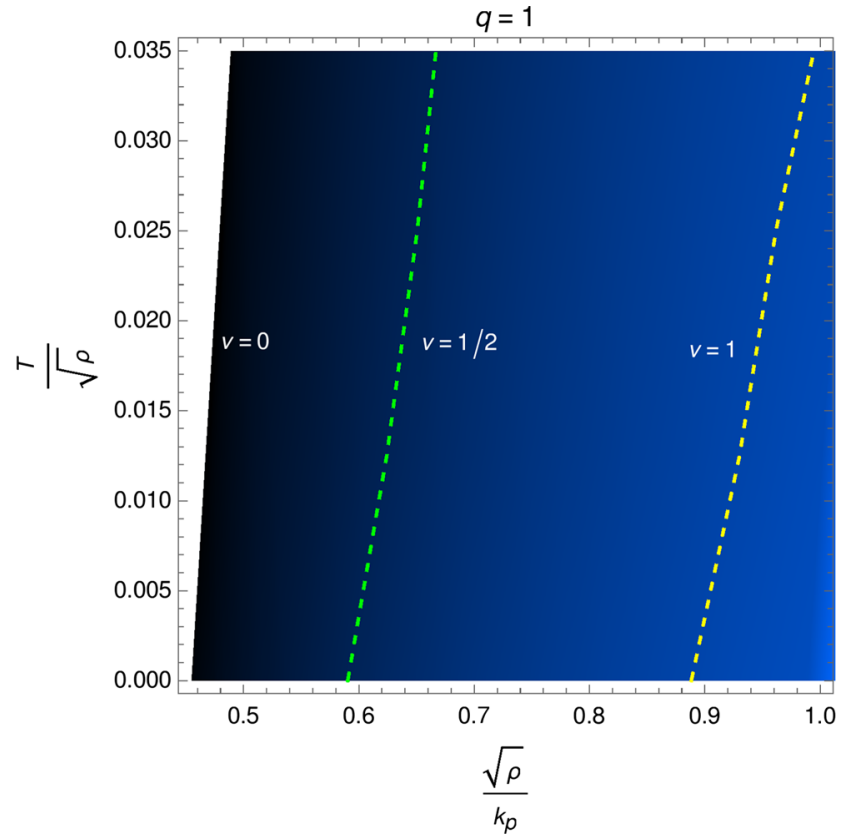

(b) $T / \sqrt{\rho}$ vs. $\sqrt{\rho} / k_{p}$ plane for $q=1$.

and gradually increase with the lighter shade. The green and yellow dashed lines correspond to $v=1 / 2$ and $v=1$ respectively

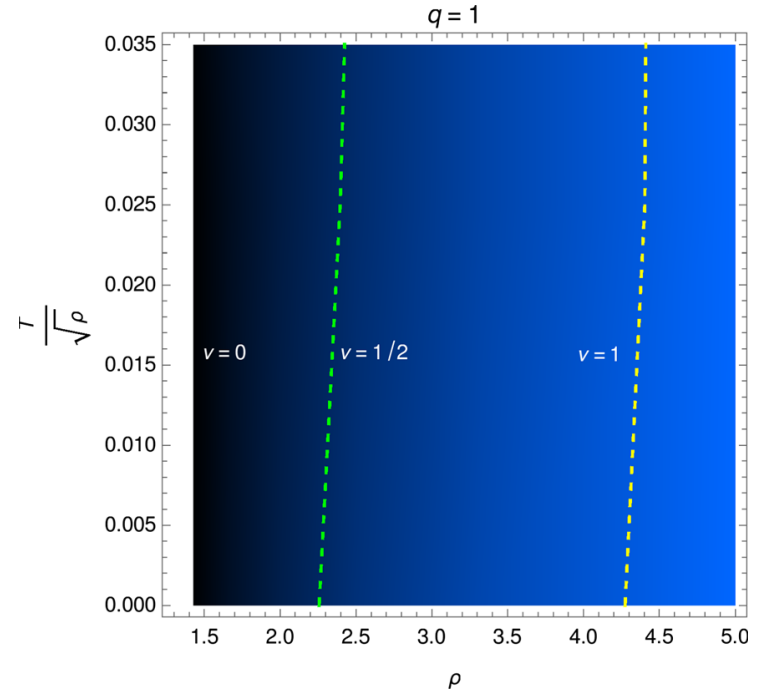

(a) $T / \sqrt{\rho}$ vs. $\rho$ plane for $q=1$.

Fig. 6 Left: Density plot of $v$ in $T / \sqrt{\rho}$ vs. $\rho$ plane. The colour gradient corresponds to the value of $v$ which increases from left to right. The white portion is the oscillatory region with darkest shades being $v=0$ and gradually increase with the lighter shade. The green and yel-

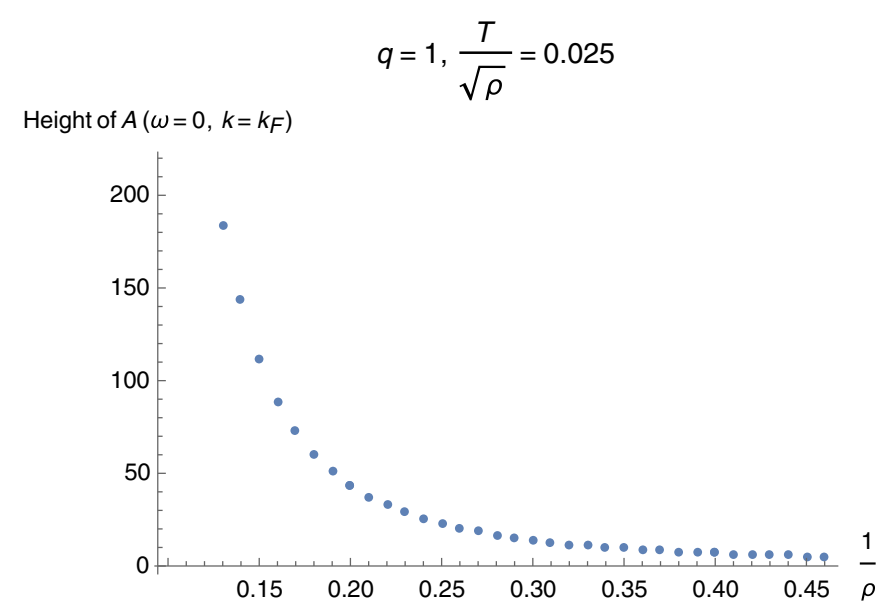

(b) Height of $A\left(\omega=0, k=k_{F}\right)$ vs. $1 / \rho$ for $q=1$ and $T / \sqrt{\rho}=0.025$.

low dashed lines correspond to $v=1 / 2$ and $v=1$ respectively. Right: Height of the spectral function decreases exponentially as the system move from Fermi toward the non-Fermi region 


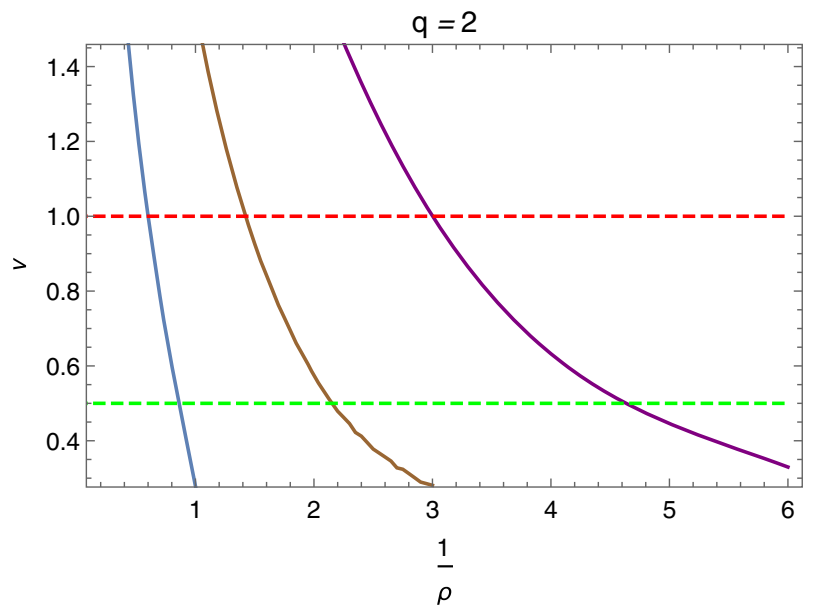

(a) $\nu$ vs. $1 / \rho$ for $q=2$ and $T=0$.

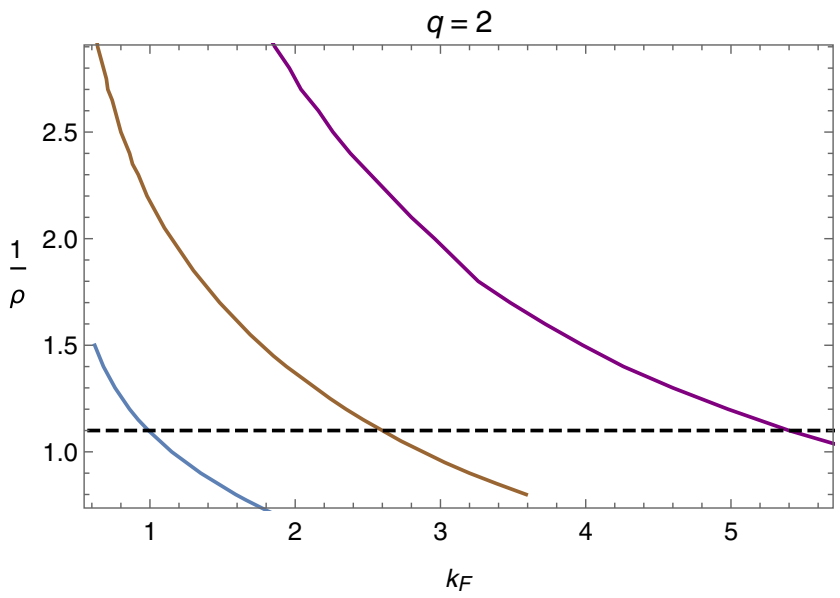

(b) $k_{F}$ vs. $1 / \rho$ for $q=2$ and $T=0$.

Fig. 7 Blue, brown and purple curve represents $Z_{2}=1,0.5$ and 0.25 respectively. Left: Red and green dashed line correspond to $v=1 / 2$ and $v=1$ line respectively. Right: Black dashed line correspond to $\rho=0.91$

Table 1 Fermi momenta $k_{F}$ and $v$ for different values of $\mathrm{f}$ parameter $Z_{2}$ with $q=2$ and $\rho=0.91$

\begin{tabular}{llll}
\hline$Z_{2}$ & 0.25 & 0.5 & 1 \\
\hline$k_{F}$ & 5.4 & 2.6 & 0.99 \\
$v$ & 3.13 & 1.39 & 0.103 \\
\hline
\end{tabular}

metal to normal metal phase increases with the increase in temperature. It may be worth mentioning that, as the system moved from the Fermi to the non-Fermi region, the height of the peak of the spectral function decreased exponentially. This can be observed from Fig. 6b, where we have plotted height of the spectral function $\left(A\left(\omega=0, k=k_{p}\right)\right)$ vs. $\rho$ for $q=1$ at $T / \rho=0.025$, with the blue dots corresponding to the Fermi momenta.

In order to see the role played by the arcsin parameter $Z_{2}$ in the fermionic behaviour, in addition to $Z_{2}=1$ we have also considered $Z_{2}=0.25$ and 0.5 , where we have chosen $T=0, q=2$ and $z_{1}=1$. In Fig. 7 a we have plotted $1 / \rho$ vs. $v$ for different values of $Z_{2}$, with blue, brown and purple curve showing $Z_{2}=1,0.5$ and 0.25 respectively, with red and green dashed line corresponding to $v=1 / 2$ and $v=1$ line respectively. We can see from this Fig. 7a that with an increase of $Z_{2}$ the transition point from Fermi to Non-Fermi liquid regime changes, for higher value of $Z_{2}$ the transition occurs at higher value of charge density $\rho$. From the Fig. 7 a or more explicitly from Table 1 , one may also observe that for a given value of $\rho$ there is a transition from Fermi to Non-Fermi liquid with an increase of arcsin parameter $Z_{2}$. We have also plotted Fermi momenta $k_{F}$ vs. inverse of charge density $1 / \rho$ in Fig. 7b, with blue, brown and purple curve representing $Z_{2}=1,0.5$ and 0.25 respectively, with black dashed line showing $\rho=0.91$. From this Fig. 7b we can see that for a given value of $\rho$ the Fermi momenta $k_{F}$ decreases with the increase of arcsin parameter $Z_{2}$ as given in Table 1. A similar kind of behaviour has been observed in [36], where the Fermi momenta increases with the decrease of BI parameter and the system which is already in the nonFermi liquid regime becomes "more non Fermi".

\subsection{Probe geometry}

In this background, we will follow a strategy similar to the one mentioned in the previous section of finite temperature. We will set $p=0$ and study the massless fermionic spectrum in finite temperature with $q=2$, where the temperature is controlled by the position of the horizon $\left(r_{h}\right)$. In this background, we will move our UV to $r \rightarrow 0$ by considering the transformation $r \rightarrow 1 / r$. In this regards, we will consider the condition given in (2.19) $z>2$ and $\theta<2$. We will set $\lambda=0, \alpha=1$ and $z=2.2$ which in turn determines the value of other parameters for cuprates like scaling given in (2.20).

We have plotted spectral function $A(\omega=0, k)$ vs $k$ for $\rho=1$ in Fig. 8a where we find a very sharp peak at $k_{F}=$ -6.305 , which is confirmed as the existence of Fermi surface from $A\left(\omega, k_{F}\right)$ vs $k$ plot given in Fig. $8 \mathrm{~b}$ where we again see a very sharp peak at $\omega=0$ with $k=k_{F}$.

Our analysis of probe limit at finite temperature shows existence of distinct Fermi surface. On comparison with the result of fully backreacted geometry in finite temperature, it can be observed that the peak is much sharper for the probe limit, which may be due to fact that the hyperscaling violating geometry leads to sharper peak. On the other hand, comparing the result with [40] where they have considered a linear Maxwell electrodynamics with the same background of hyperscaling violating geometry as used in our probe limit, we find that the present analysis leads to much sharper peak. 


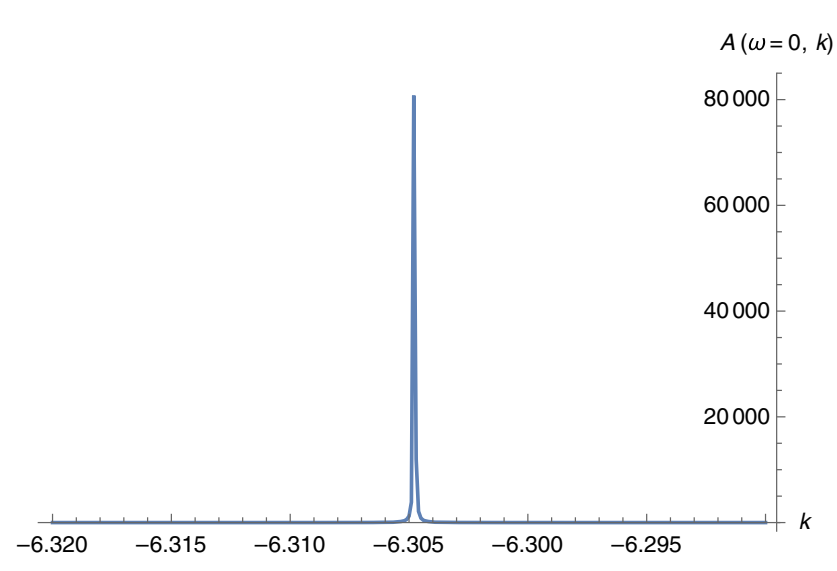

(a) $A(\omega=0, k)$ vs. $k$ for $q=2, \rho=1$.

Fig. $8 A(\omega, k)$ vs. $k$ and $\omega$ with $q=2, \rho=1$

In this case, one can attribute sharpness of the peak to the non-linear electrodynamics considered in this work.

\section{Discussion}

We have studied the fermionic behaviour of the nonlinear arcsin electrodynamics model given in [35]. We have evaluated the spectral function to determine the existence of a Fermi surface in zero and finite temperature. In case of zero temperature, we find that for a given value of charge density $\rho$ the particle with higher charge $q$ has a higher chance of forming a Fermi surface. For a given charge of the particle $q$, the system with large charge density $\rho$ is in the region of normal metallic phase and on decreasing the charge density the system starts to move toward the marginal Fermi liquid regime where $v=1 / 2$. On the further reduction of the charge density $\rho$ results in the transition/crossover toward the strange metal phase. The system shows a similar kind of behaviour with the variation of the momentum dissipation $k_{p}$, where there is a transition/crossover from normal metal to strange metal phase, which may be considered as a disorder driven transition [32].

Next, we investigated the finite temperature case, where we see for a given charge $q$ with the increase in temperature the height of the spectral function $A(\omega, k)$ starts decreasing and becomes broader and broader, showing that as the temperature rises the density gets spread out due to the thermal excitation. We have also studied the nature of the Fermi surface with the variation of momentum dissipation $k_{p}$ and charge density of the system $\rho$ for finite temperature case. We see that with an increase in temperature the critical value of momentum dissipation for the transition decreases. In case of variation with the charge density $\rho$, with increase in temperature the critical value of charge density for the transition

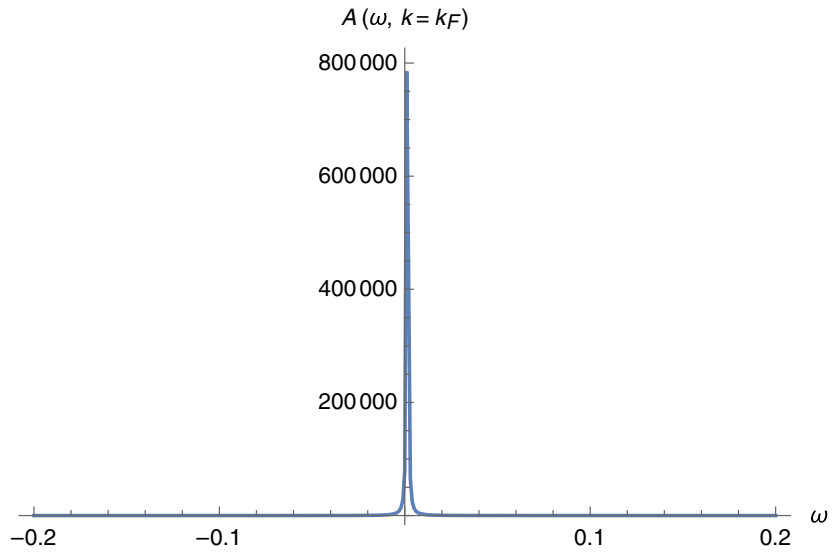

(b) $A\left(\omega, k=k_{F}\right)$ vs. $\omega$ for $q=2, \rho=1$.

increases. For a given value of momentum dissipation and charge density with the increase in temperature, there is a transition from normal metal to strange metal phase, which is similar to the case in cuprates high $T_{c}$ superconductors where the transition depends on the doping.

In the present model as already mentioned in the introduction, we have considered a particular phase of the dual boundary theory. Comparing these results with the results of [39], we see that the boundary charge density $\rho$ plays an important role in both the phases of the dual boundary theory. In the case of [39], $\rho$ determines the critical phase transition temperature of the superconductor and also in the present case $\rho$ determines the critical transition temperature from the regime of Fermi to Non-Fermi. In both cases, the transition temperature increases with the increase of charge density $\rho$. In particular, we have observed that in [39] for a given temperature as $\beta$ increases the critical value of charge density increases similarly in the present model if we keep the temperature fixed at $T=0$ and increase the analogous parameter $Z_{2}$ the critical value of charge density also increases. We have also studied the fermionic behaviour with the variation of the arcsin parameter $Z_{2}$, where we find that with the increase of the arcsin parameter there is a transition from Fermi to nonFermi regime. We have also analysed the behaviour of Fermi momenta $k_{F}$ with the variation of the $\arcsin$ parameter $Z_{2}$, where we find that for a given boundary charge density $\rho$, Fermi momenta decreases with the increase of arcsin parameter. Comparing this result with another model of nonlinear electrodynamics studied in [36], where the nonlinearity was controlled by BI parameter, we see that in both the cases with the increase of nonlinear parameter the Fermi momenta decreases and the system which is already in the non-Fermi regime moves toward "more non-Fermi" regime.

We have also considered the probe limit of this nonlinear system with hyperscaling violating geometry as the back- 
ground. It was shown in $[33,35]$ that with the particular choices of the parameters in this background, the system exhibits a linear temperature dependence of resistivity as in the case of cuprates. We have chosen those particular parameters and studied the fermionic spectrum in a finite temperature, where we find a very sharp peak in the spectral function indicating the existence of the Fermi surface. Comparing these results with [40], where linear Maxwell electrodynamics was considered in the same background as our probe background, we find that the height of the peak in the our case is much higher and sharper. This feature can be attributed to non-linear electrodynamics.

In this work, we have chosen the neutral scalar field $\phi$ to vanish in the case of backreacted geometry and consider a simpler solution. Considering more general configurations with non-zero neutral scalar $\phi$ along with non-trivial choices of the coupling $Z_{1}$ may lead to further interesting solution and it would be interesting to study the fermionic behaviour for such backgrounds. It has been shown in [35] that by turning on the magnetic field $h$ may give rise to metal-insulator transition with a variation of $h$. Analysis of fermionic excitations in presence of magnetic field may lead to further insight on such transitions. In [39], the author have analysed the superconducting phase of this nonlinear electrodynamic model in finite temperature. It may be interesting to study the fermionic behaviour in this superconducting phase at finite temperature as well as its zero temperature limit which may correspond to a domain wall solutions along the line of [41-43]. As we have mentioned the dual boundary theory associated with this model admits a rich phase structure and it would be interesting to incorporate all of these phases in a single gravity theory. Another natural extension of the present work is to consider $U(1) \times U(1)$ gauge fields in this setup and introduce doping along the line of [22]. We would like to report on some of these in future.

Acknowledgements I would like to acknowledge Subir Mukhopadhyay for his valuable suggestions and help throughout the work.

Data Availability Statement This manuscript has no associated data or the data will not be deposited. [Authors' comment: The datasets generated during the current study available from the corresponding author nishalrai10@gmail.com on reasonable request.]

Open Access This article is distributed under the terms of the Creative Commons Attribution 4.0 International License (http://creativecomm ons.org/licenses/by/4.0/), which permits unrestricted use, distribution, and reproduction in any medium, provided you give appropriate credit to the original author(s) and the source, provide a link to the Creative Commons license, and indicate if changes were made. Funded by SCOAP ${ }^{3}$.

\section{References}

1. S.A. Hartnoll, Class. Quant. Grav. 26, 224002 (2009). https://doi. org/10.1088/0264-9381/26/22/224002. arXiv:0903.3246 [hep-th]
2. C.P. Herzog, J. Phys. A 42, 343001 (2009). https://doi.org/10.1088/ 1751-8113/42/34/343001. arXiv:0904.1975 [hep-th]

3. G.T. Horowitz, Lect. Notes Phys. 828, 313 (2011). https://doi.org/ 10.1007/978-3-642-04864-7-10. arXiv:1002.1722 [hep-th]

4. G.T. Horowitz, M.M. Roberts, Phys. Rev. D 78, 126008 (2008). https://doi.org/10.1103/PhysRevD.78.126008. arXiv:0810.1077 [hep-th]

5. S.A. Hartnoll arXiv:1106.4324 [hep-th]

6. S. Sachdev, Ann. Rev. Condensed Matter Phys. 3, 9 (2012). https://doi.org/10.1146/annurev-conmatphys-020911-125141. arXiv:1108.1197 [cond-mat.str-el]

7. A.G. Green, Contemp. Phys. 54(1), 33 (2013). https://doi.org/10. 1080/00107514.2013.779477. arXiv:1304.5908 [cond-mat.str-el]

8. S.A. Hartnoll, A. Lucas, S. Sachdev, arXiv:1612.07324 [hep-th]

9. J.M. Maldacena, Int. J. Theor. Phys. 38, 1113 (1999)

10. J. M. Maldacena, Adv. Theor. Math. Phys. 2, 231 (1998). https:// doi.org/10.1023/A:1026654312961[hep-th/9711200]

11. S.S. Gubser, I.R. Klebanov, A.M. Polyakov, Phys. Lett. B 428, 105 (1998). https://doi.org/10.1016/S0370-2693(98)00377-3. arXiv:hep-th/9802109

12. E. Witten, Adv. Theor. Math. Phys. 2, 253 (1998). arXiv:hep-th/9802150

13. S.S. Lee, Phys. Rev. D 79, 086006 (2009). https://doi.org/10.1103/ PhysRevD.79.086006. arXiv:0809.3402 [hep-th]

14. M. Cubrovic, J. Zaanen, K. Schalm, Science 325, 439 (2009). https://doi.org/10.1126/science.1174962. arXiv:0904.1993 [hepth]

15. H. Liu, J. McGreevy, D. Vegh, Phys. Rev. D 83, 065029 (2011). https://doi.org/10.1103/PhysRevD.83.065029. arXiv:0903.2477 [hep-th]

16. T. Faulkner, H. Liu, J. McGreevy, D. Vegh, Phys. Rev. D 83, 125002 (2011). https://doi.org/10.1103/PhysRevD.83.125002. arXiv:0907.2694 [hep-th]

17. M. Edalati, R.G. Leigh, K.W. Lo, P.W. Phillips, Phys. Rev. D 83, 046012 (2011). https://doi.org/10.1103/PhysRevD.83.046012. arXiv:1012.3751 [hep-th]

18. M. Edalati, R.G. Leigh, P.W. Phillips, Phys. Rev. Lett. 106, 091602 (2011). https://doi.org/10.1103/PhysRevLett.106.091602. arXiv: 1010.3238 [hep-th]

19. J.P. Wu, JHEP 1304, 073 (2013). https://doi.org/10.1007/ JHEP04(2013)073

20. J.P. Wu, Phys. Lett. B 728, 450 (2014). https://doi.org/10.1016/j. physletb.2013.11.040

21. L.Q. Fang, X.M. Kuang, B. Wang, J.P. Wu, JHEP 1511, 134 (2015). https://doi.org/10.1007/JHEP11(2015)134. arXiv:1507.03121 [hep-th]

22. G. Giordano, N. Grandi, A. Lugo, R. Soto-Garrido, JHEP 1810, 068 (2018). https://doi.org/10.1007/JHEP10(2018)068. arXiv:1808.02145 [hep-th]

23. O. DeWolfe, S.S. Gubser, C. Rosen, Phys. Rev. Lett. 108, 251601 (2012). https://doi.org/10.1103/PhysRevLett.108.251601. arXiv:1112.3036 [hep-th]

24. O. DeWolfe, S.S. Gubser, C. Rosen, Phys. Rev. D 86, 106002 (2012). https://doi.org/10.1103/PhysRevD.86.106002. arXiv:1207.3352 [hep-th]

25. O. DeWolfe, O. Henriksson, C. Rosen, Phys. Rev. D 91(12), 126017 (2015). https://doi.org/10.1103/PhysRevD.91.126017. [arXiv:1410.6986 [hep-th]]

26. S. Mukhopadhyay, N. Rai, Phys. Rev. D 96(2), 026005 (2017). https://doi.org/10.1103/PhysRevD.96.026005

27. C. Cosnier-Horeau, S.S. Gubser, Phys. Rev. D 91(6), 066002 (2015). https://doi.org/10.1103/PhysRevD.91.066002. [arXiv: 1411.5384 [hep-th]]

28. S. Mukhopadhyay, N. Rai, Phys. Rev. D 96(6), 066001 (2017). https://doi.org/10.1103/PhysRevD.96.066001 
29. E. Kiritsis, L. Li, J. Phys. A 50(11), 115402 (2017). https:// doi.org/10.1088/1751-8121/aa59c6. [arXiv:1608.02598 [condmat.str-el]]

30. A. Karch, A. O'Bannon, JHEP 0709, 024 (2007). https://doi.org/ 10.1088/1126-6708/2007/09/024. arXiv:0705.3870 [hep-th]

31. A. O'Bannon, Phys. Rev. D 76, 086007 (2007). https://doi.org/10. 1103/PhysRevD.76.086007. arXiv:0708.1994 [hep-th]

32. S. Cremonini, A. Hoover, L. Li, JHEP 1710, 133 (2017). https:// doi.org/10.1007/JHEP10(2017).133. arXiv:1707.01505 [hep-th]

33. E. Blauvelt, S. Cremonini, A. Hoover, L. Li, S. Waskie, Phys. Rev. D 97(6), 061901 (2018). https://doi.org/10.1103/PhysRevD. 97.061901. arXiv:1710.01326 [hep-th]

34. S. Cremonini, A. Hoover, L. Li, S. Waskie, Phys. Rev. D 99(6), 061901 (2019). https://doi.org/10.1103/PhysRevD.99. 061901. [arXiv:1812.01040 [hep-th]]

35. N. Rai, S. Mukhopadhyay, DC conductivity from backreacted nonlinear arcsin electrodynamics. Ann. Phys. (2019). https://doi.org/ 10.1016/j.aop.2019.167974

36. J.P. Wu, Phys. Lett. B 758, 440 (2016). https://doi.org/10.1016/j. physletb.2016.05.049. arXiv:1705.06707 [hep-th]
37. S.I. Kruglov, Annalen Phys. 527, 397 (2015). https://doi.org/10. 1002/andp.201500142. arXiv:1410.7633 [physics.gen-ph]

38. S.I. Kruglov, Commun. Theor. Phys. 66(1), 59 (2016). https://doi. org/10.1088/0253-6102/66/1/059. arXiv:1511.03303 [hep-ph]

39. S.I. Kruglov, Annalen Phys. 530(8), 1800070 (2018). https://doi. org/10.1002/andp.201800070. arXiv:1801.06905 [hep-th]

40. X.M. Kuang, E. Papantonopoulos, B. Wang, J.P. Wu, JHEP 1411, 086 (2014). https://doi.org/10.1007/JHEP11(2014).086. arXiv:1409.2945 [hep-th]

41. S. Mukhopadhyay, N. Rai, Phys. Rev. D 98(2), 026007 (2018). https://doi.org/10.1103/PhysRevD.98.026007. arXiv:1904.06147 [hep-th]

42. S.S. Gubser, S.S. Pufu, F.D. Rocha, Phys. Lett. B 683, 201 (2010). https://doi.org/10.1016/j.physletb.2009.12.017. arXiv:0908.0011 [hep-th]

43. S. Mukhopadhyay, N. Rai, Phys. Lett. B 780, 608 (2018). https:// doi.org/10.1016/j.physletb.2018.03.037 